\title{
PERSISTENT RANDOM WALKS MAY HAVE ARBITRARILY LARGE TAILS
}

\author{
D. R. GREY*, University of Sheffield
}

\begin{abstract}
We give a probabilistic proof of a result of Shepp, that a symmetric random walk may have jump size distribution with arbitrarily large tails and yet still be persistent.
\end{abstract}

PERSISTENCE; TRANSIENCE; TAIL BEHAVIOUR

\section{Introduction and statement of result}

Let $X_{1}, X_{2}, \cdots$ be independent, identically distributed random variables, which we shall take to be integer valued and symmetrically distributed about zero. Define the random walk $\left\{S_{n}\right\}$ by

$$
S_{n}=X_{1}+X_{2}+\cdots+X_{n} \text { for } n=0,1,2, \cdots \text {. }
$$

The random walk is called persistent if

$$
P\left(S_{n}=0 \text { for some } n \geqq 1\right)=1 .
$$

Otherwise it is called transient. The one-dimensional Chung-Fuchs theorem (see Section 3) tells us that for the random walk to be transient it is necessary that $E\left|X_{1}\right|=\infty$. If the tail $G(x)=P\left(\left|X_{1}\right|>x\right)$ is assumed to be regularly varying, then using the analytic necessary and sufficient condition for persistence (Spitzer (1964)) and Tauberian theorems of Pitman (1968) it is possible to obtain necessary and sufficient conditions in terms of $G$. However, Shepp (1964) proved the following surprising result.

Theorem. Given a function $\varepsilon$ with $\varepsilon(x) \downarrow 0$ as $x \rightarrow \infty$, there exists a persistent random walk with $G(x) \geqq \varepsilon(x)$ for all large enough $x$.

This result suggests that the size of the tail of the distribution of $X_{1}$ does not tell the whole story. It may be regarded as a counterexample in the spirit of Stoyanov (1987).

The purpose of this note is to provide a probabilistic proof of Shepp's theorem, which was originally proved by analytic methods involving characteristic functions. The motivation for considering this problem was the resemblance to a similar problem in branching processes (Grey (1989)).

\section{A probabilistic proof}

Let $\left(p_{1}, p_{2} \cdots\right)$ be a probability distribution with $p_{i}>0$ for all $i=1,2, \cdots$, and let $\left(x_{1}, x_{2}, \cdots\right)$ be an increasing sequence of positive integers. Let $W_{k}$ be the defective random walk which takes jumps of sizes $\pm x_{i}$ with probabilities $\frac{1}{2} p_{i}$ for $i=1,2, \cdots, k$, and which 'dies' at any step with probability $q_{k}=1-\sum_{i=1}^{k} p_{i}$. We show below that it is possible to choose the pairs $\left(p_{1}, x_{1}\right),\left(p_{2}, x_{2}\right), \cdots$ successively, in the style of Grey $(1978)$, in such a way that

$$
W_{k} \text { has probability at least } 1-k^{-1} \text { of returning to zero before dying; }
$$

Received 26 August 1988; revision received 15 November 1988.

* Postal address: Department of Probability and Statistics, The University, Sheffield S3 7RH, UK. 
and

$$
q_{k} \geqq \varepsilon\left(x_{k}\right) .
$$

It then follows, if $W_{1}, W_{2}, \cdots$ are constructed on the same probability space in an obvious way, that $W=\lim _{k \rightarrow \infty} W_{k}$ exists in a strong sense, is a proper random walk taking jumps of sizes $\pm x_{i}$ with probabilities $\frac{1}{2} p_{i}$ for $i=1,2, \cdots$, and, because of (1) and (2), satisfies the requirements of the theorem.

The proof is by induction. The choice of $\left(p_{1}, x_{1}\right)$ poses no problem. Suppose, therefore, that we have chosen $\left(p_{1}, x_{1}\right), \cdots,\left(p_{k}, x_{k}\right)$. Consider the proper random walk on the two-dimensional integer lattice $\mathbb{Z}^{2}$ taking jumps of sizes $\pm\left(x_{i}, 0\right)$ with probabilities $\frac{1}{2} p_{i}$ and jumps of sizes $\pm(0,1)$ each with probability $\frac{1}{2} q_{k}$. This random walk is persistent, by the two-dimensional Chung-Fuchs theorem (see Section 3). Hence it has countably many partial sample paths returning to $(0,0)$ and with total probability 1 . If we reduce the probability of jumps of sizes $\pm(0,1)$ by a suitably small amount to $\frac{1}{2} p_{k+1}$ say, thereby making the random walk defective, we can ensure that these partial sample paths still have total probability at least $1-(k+1)^{-1}$. If we now choose $x_{k+1}>x_{k}$ such that $\varepsilon\left(x_{k+1}\right) \leqq q_{k+1}$ where $q_{k+1}=1-\sum_{i=1}^{k+1} p_{i}$, and map this defective random walk linearly into one dimension using the transformation $(x, y) \rightarrow x+y x_{k+1}$, we have constructed $W_{k+1}$. This completes the induction.

\section{The Chung-Fuchs theorem}

The above proof depends upon the following result, which we state for completeness. Here $\|\cdot\|$ denotes Euclidean length.

Theorem. (Chung and Fuchs (1951); see also Chung and Ornstein (1962)).

(a) If $E X_{1}=0$ then the random walk $\left\{S_{n}\right\}$ in one dimension is persistent.

(b) If $E X_{1}=0$ and $E\left\|X_{1}\right\|^{2}<\infty$ then the random walk $\left\{S_{n}\right\}$ in two dimensions is persistent.

\section{Acknowledgement}

I should like to thank Professor G. E. H. Reuter for drawing to my attention the result of Shepp (1964).

\section{References}

ChUNG, K. L. AND Fuchs, W. H. J. (1951) On the distribution of values of sums of random variables. Mem. Amer. Math. Soc. No. 6.

Chung, K. L. AND ORnStein, D. (1962) On the recurrence of sums of random variables. Bull. Amer. Math. Soc. 68, 30-32.

GREY, D. R. (1978) Three lemmas after Besicovitch and their applications in probability theory. $J$. London Math. Soc. (2) 18, 173-180.

Grey, D. R. (1989) A note on explosiveness of Markov branching processes. Adv. Appl. Prob. 21, 226-228.

PItMAN, E. J. G. (1968) On the behaviour of the characteristic function of a probability distribution in the neighbourhood of the origin. J. Austral. Math. Soc. 8, 423-443.

SHEPP, L. A. (1964) Recurrent random walks with arbitrarily large steps. Bull. Amer. Math. Soc. 70, $540-542$.

Spitzer, F. (1964) Principles of Random Walk. Van Nostrand, Princeton, N.J.

Stoyanov, J. M. (1987) Counterexamples in Probability. Wiley, Chichester. 